\title{
Dilepton production from resonance scattering in hot hadronic matter
}

\author{
Chungsik Song and C. M. Ko \\ Cyclotron Institute and Physics Department, Texas A\&M University, College Station, Texas 77843
}

(Received 8 November 1995)

\begin{abstract}
Dilepton production from resonance scattering in hot hadronic matter is studied. Including the widths of these resonances, which enhance the phase space for dilepton production, we find that the production rate is significantly increased if a resonance appears in the extended phase space. For the reaction $\pi+\rho \rightarrow l^{+}+l^{-}$, the finite $\rho$ meson width extends the invariant mass below the $\omega$-meson mass, so a peak at the $\omega$-meson mass is seen in the dilepton spectrum. Similarly, a $\rho$-meson peak appears in the reaction $\rho+\rho \rightarrow l^{+}+l^{-}$. On the other hand, the effect of particle widths in the reaction $\pi+a_{1} \rightarrow l^{+}+l^{-}$is small since the extended phase space does not include any resonance. [S0556-2813(96)02105-X]
\end{abstract}

PACS number(s): 25.75.Gz, 12.38.Mh, 21.65.+f, 24.85.+p

\section{INTRODUCTION}

At very high temperature and/or density, as achieved in ultrarelativistic heavy ion collisions [1], the hadronic matter is expected to undergo a phase transition to the quark-gluon plasma in which quarks and gluons interact weakly and the spontaneously broken chiral symmetry is restored [2]. One possible way to verify the existence of the quark-gluon plasma in these collisions is to detect the produced photons and dileptons. Since these electromagnetically interacting particles have mean free paths much larger than the transverse size of the colliding system, they leave the system without further interactions and thus carry information about the hot dense matter in which they are produced $[3,4]$.

However, photons and dileptons can also be produced from hadronic matter below the phase transition temperature and density. To find the signals for the quark-gluon plasma thus needs a good understanding of this background contribution. On the other hand, dilepton production from hadronic matter is useful for understanding precursor phenomena, such as the change of meson masses and widths, related to chiral symmetry restoration and the deconfinement phase transition in hot dense hadronic matter [5-8].

Recently, photon [9-12] and dilepton [13,14] production from hadronic matter have been extensively studied with the effective Lagrangian. It has been shown that in hadronic matter reactions with $a_{1}$ meson in the intermediate state, e.g., $\pi+\rho \rightarrow a_{1} \rightarrow \pi+\gamma$, significantly enhance the photon production rate. Also, for dileptons with invariant masses in the region 1.5-3.0 MeV, which has been suggested as a possible window for observing dileptons from the quark-gluon plasma, contributions from resonance scattering, such as $\pi+\rho \rightarrow l^{+}+l^{-}, \rho+\rho \rightarrow l^{+}+l^{-}, \pi+a_{1} \rightarrow l^{+}+l^{-}$, etc., are important. Recent experimental data from CERN/SPS experiments seem to be consistent with these observations $[15,16]$.

In previous studies, some important features of hadron in-medium properties have not been included. The present authors and Lee [17] have shown that the pion electromagnetic form factor is reduced at finite temperature, leading thus to a suppression of dilepton production from pion-pion annihilation. The reduction of the from factor at finite temperature, which can be related to the deconfinement transi- tion and chiral symmetry restoration in hot hadronic matter, has also been shown in calculations based on the QCD sum rule at finite temperature [18] and the QCD factorization assumption at large momentum transfer [19].

Another feature, which is the subject of the present paper, is that most mesons are not stable and easily decay into other mesons. The widths of these mesons are not negligible even in free space, e.g., $\Gamma_{\rho} \sim 150 \mathrm{MeV}$ and $\Gamma_{a_{1}} \sim 400 \mathrm{MeV}$ for the $\rho$ and $a_{1}$ mesons, respectively, and, furthermore, they are also expected to change with temperature. The finite particle width increases the phase space for dilepton production and thus modifies its production rate from the hadronic matter. In this work, we shall calculate the dilepton production rate from hot hadronic matter by including the particle widths. This study can be easily extended to photon production from hot matter.

\section{FORMALISM}

We consider dilepton production from a reaction in which two hadrons $\left(h_{1}, h_{2}\right)$ annihilate into a pair of leptons through a resonance $\left(r^{*}\right)$, i.e., $h_{1}+h_{2} \rightarrow r^{*} \rightarrow l^{+}+l^{-}$, as shown in Fig. 1. The dilepton production rate $(R) /$ volume $(V)$ from such a reaction in hot matter can be written as [4]

$$
\begin{aligned}
\frac{R}{V}= & -4 e^{4} \int \frac{d^{3} \mathbf{k}_{1}}{(2 \pi)^{3} 2 \omega_{1}} \frac{d^{3} \mathbf{k}_{2}}{(2 \pi)^{3} 2 \omega_{2}} \frac{d^{3} \mathbf{p}_{1}}{(2 \pi)^{3} 2 E_{1}} \frac{d^{3} \mathbf{p}_{2}}{(2 \pi)^{3} 2 E_{2}} \\
& \times(2 \pi)^{4} \delta^{(4)}\left(k_{1}+k_{2}-p_{1}-p_{2}\right) f\left(\omega_{1}\right) f\left(\omega_{2}\right) l^{\mu \nu}\left(p_{1}, p_{2}\right) \\
& \times\left(\frac{1}{q^{2}}\right)^{2} h_{\mu \nu}\left(k_{1}, k_{2}\right) .
\end{aligned}
$$

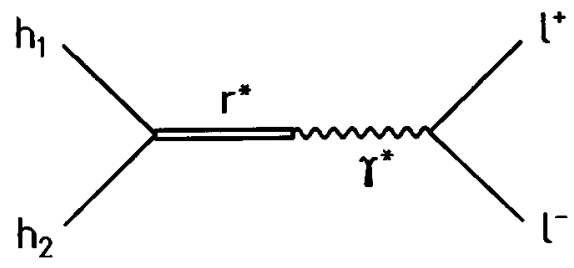

FIG. 1. Dilepton production from hadron scattering. 
In the above $k_{i}=\left(\omega_{i}, \mathbf{k}_{i}\right)$ and $p_{i}=\left(E_{i}, \mathbf{p}_{i}\right)$ are, respectively, the four-momenta of the colliding hadrons and dileptons, and $q=p_{1}-p_{2} ; f(\omega)$ is the Fermi-Dirac or Bose-Einstein distribution function; $h_{\mu \nu}\left(k_{1}, k_{2}\right)$ is the hadronic tensor which depends on the reaction; and the leptonic tensor $l^{\mu \nu}\left(p_{1}, p_{2}\right)$ is given by

$$
l^{\mu \nu}\left(p_{1}, p_{2}\right)=\left(p_{1} \cdot p_{2}+m_{l}^{2}\right) g^{\mu \nu}-p_{1}^{\mu} p_{2}^{\nu}-p_{1}^{\nu} p_{2}^{\mu} .
$$

In Eq. (1) it has been assumed that the colliding particles are on mass shell, i.e., $\omega_{i}=\sqrt{\mathbf{k}_{i}^{2}+m_{i}^{2}}$. However, these particles in general have finite widths and should be described by spectral functions $\rho_{i}\left(\omega_{i}, \mathbf{k}_{i}\right)$. This requires the following modification [20]:

$$
\int \frac{d^{3} \mathbf{k}}{(2 \pi)^{3} 2 \omega} \rightarrow \int \frac{d^{3} \mathbf{k}}{(2 \pi)^{3}} \int \frac{d \omega^{\prime}}{(2 \pi)} \rho\left(\omega^{\prime}, \mathbf{k}\right),
$$

and leads to

$$
\begin{aligned}
\frac{d(R / V)}{d M^{2}}= & -4 e^{4} \int \frac{d^{4} k_{1}}{(2 \pi)^{4}} \frac{d^{4} k_{2}}{(2 \pi)^{4}} \rho_{1}\left(\omega_{1}, \mathbf{k}_{1}\right) \rho_{2}\left(\omega_{2}, \mathbf{k}_{2}\right) \\
& \times f\left(\omega_{1}\right) f\left(\omega_{2}\right) h_{\mu \nu}\left(k_{1}, k_{2}\right) L^{\mu \nu}\left(k_{1}, k_{2}, M^{2}\right),
\end{aligned}
$$

where $M$ is the invariant mass of the dilepton, $M^{2}=\left(p_{1}+p_{2}\right)^{2}$, and $L_{\mu \nu}\left(k_{1}, k_{2}, M^{2}\right)$ is given by

$$
\begin{aligned}
L_{\mu \nu}\left(k_{1}, k_{2}, M^{2}\right)= & \int \frac{d^{3} \mathbf{p}_{1}}{(2 \pi)^{3} 2 E_{1}} \int \frac{d^{3} \mathbf{p}_{2}}{(2 \pi)^{3} 2 E_{2}}(2 \pi)^{4} \delta^{(4)} \\
& \times\left(k_{1}+k_{2}-p_{1}-p_{2}\right) \delta\left[\left(p_{1}+p_{2}\right)^{2}-M^{2}\right] \\
& \times l_{\mu \nu}\left(p_{1}, p_{2}\right)\left(\frac{1}{q^{2}}\right)^{2}
\end{aligned}
$$

For dileptons at rest in the center-of-momentum frame, this can be written as

$$
\left.\frac{d L^{\mu \nu}}{d^{3} \mathbf{q}}\right|_{\mathbf{q}=0}=\frac{1}{24(2 \pi) M^{3}} \delta\left(K_{0}-M\right) \delta^{(3)}(\mathbf{K})\left(g^{\mu \nu}-\frac{Q^{\mu} Q^{\nu}}{M^{2}}\right),
$$

with $Q=(M, 0,0,0)$ and $\left(K_{0}, \mathbf{K}\right)=\left(\omega_{1}+\omega_{2}, \mathbf{k}_{1}+\mathbf{k}_{2}\right)$.

From Eqs. (4) and (6), we have

$$
\begin{aligned}
\left.\frac{d(R / V)}{d M d^{3} \mathbf{q}}\right|_{\mathbf{q}=0}= & \frac{e^{4}}{6(2 \pi)^{9} M^{3}} \int d^{3} \mathbf{k}_{1} d w_{1} \rho_{1}\left(\omega_{1}, \mathbf{k}_{1}\right) \\
& \times \rho_{2}\left(M-\omega_{1},-\mathbf{k}_{1}\right) f_{1}\left(\omega_{1}\right) f_{2}\left(M-\omega_{1}\right) \\
& \times W\left(\omega_{1}, \mathbf{k}_{1}, M\right)
\end{aligned}
$$

with

$$
W\left(\omega_{1}, \mathbf{k}_{1}, M\right)=h^{\mu \nu}\left(k_{1}, Q-k_{1}\right)\left(-g_{\mu \nu}+\frac{Q_{\mu} Q_{\nu}}{M^{2}}\right)
$$

One can easily check that when particles are taken to be on mass shell, i.e., $\rho(\omega, \mathbf{k})=2 \pi \delta\left(k^{2}-m^{2}\right)$, Eq. (7) reduces to the one used in the literature [6].

\section{EFFECTIVE CHIRAL LAGRANGIAN}

We assume that the hadronic matter consists of pions, vector mesons, and axial vector mesons and that their interactions are described by an effective chiral Lagrangian. The interactions among these hadrons are constrained by symmetry properties of the strong interaction. The resulting parameters in the Lagrangian are then determined from the experimental data on hadron properties [21]. The chiral Lagrangian for pseudoscalar mesons at low energies takes the form

$$
\mathscr{L}=-\frac{1}{8} F_{\pi}^{2} \operatorname{Tr}\left(\partial_{\mu} U \partial^{\mu} U^{\dagger}\right)
$$

where $F_{\pi}=135 \mathrm{MeV}$ is the pion decay constant, and $U$ is related to pseudoscalar mesons $P$ by

$$
U=\exp \left[\frac{2 i}{F_{\pi}} P\right], \quad P=\pi^{a} \frac{\lambda^{a}}{\sqrt{2}} .
$$

Vector $\left(V_{\mu}\right)$ and axial vector $\left(A_{\mu}\right)$ mesons are included as massive Yang-Mills fields of the chiral symmetry [22] and the derivative in Eq. (9) is thus replaced by the covariant one, i.e.,

$$
\partial_{\mu} U \rightarrow\left(\partial_{\mu} U-i g A_{\mu}^{L} U+i g U A_{\mu}^{R}\right),
$$

where $A_{L}^{\mu}=\left(V^{\mu}-A^{\mu}\right) / 2$ and $A_{R}^{\mu}=\left(V^{\mu}+A^{\mu}\right) / 2$. The phenomenological gauge coupling constant $(g)$ can be determined by fitting the masses and widths of vector and axial vector mesons [23]. The electromagnetic interaction is then introduced through the notion of vector meson dominance (VMD) [24], i.e.,

$$
\mathscr{L}_{\mathrm{em}}=-\frac{\sqrt{2} e}{g} a^{\mu}\left[m_{\rho}^{2} \rho_{\mu}^{0}+\frac{1}{3} m_{\omega}^{2} \omega_{\mu}-\frac{\sqrt{2}}{3} m_{\phi}^{2} \phi_{\mu}\right] .
$$

We also include the gauged Wess-Zumino term in the effective Lagrangian to describe the non-Abelian anomaly structure of QCD [25], which leads to an anomalous interaction among a pseudoscalar meson and two vector mesons,

$$
\mathscr{L}_{V V P}=-\frac{3 g^{2}}{16 \pi^{2} F_{\pi}} \epsilon^{\mu \nu \alpha \beta} \operatorname{Tr}\left(\partial_{\mu} V_{\nu} \partial_{\alpha} V_{\beta} P\right),
$$

where $\epsilon^{\mu \nu \alpha \beta}$ is the antisymmetric Levi-Civita tensor with $\epsilon^{0123}=1$.

From Eq. (13) we have

$$
\mathscr{L}_{\omega \pi \rho}=-\sqrt{2} g_{\omega} \epsilon^{\mu \nu \alpha \beta} \partial_{\mu} \omega_{\nu} \partial_{\alpha} \rho_{\beta} \cdot \pi,
$$

with

$$
g_{\omega}=\left(\frac{3 g^{2}}{16 \pi^{2} F_{\pi}}\right)
$$

Combining with the vector meson dominance assumption, we obtain

$$
\Gamma\left(\omega \rightarrow \pi^{0} \gamma\right)=\frac{3}{64 \pi^{4}} \frac{\alpha g^{2}}{F_{\pi}^{2}}\left|\mathbf{q}_{\pi}\right|^{3}=0.80 \mathrm{MeV}
$$


This is in good agreement with the experimental value, $\Gamma_{\text {expt }}\left(\omega \rightarrow \pi^{0} \gamma\right)=0.86 \pm 0.05 \mathrm{MeV}$. There is another anomalous decay, $\phi \rightarrow 3 \pi$, which violates the Okubo-Zweig-Iizuka (OZI) rule. We regard this decay as being due to a small $\omega$ - $\phi$ mixing:

$$
\begin{aligned}
& \omega_{\mu}=\widetilde{\omega}_{\mu}-\epsilon \widetilde{\phi}_{\mu}, \\
& \omega_{\mu}=\epsilon \widetilde{\omega}_{\mu}+\widetilde{\phi}_{\mu},
\end{aligned}
$$

where a tilde denotes physical fields. From the anomalous $V V P$ interaction, we find

$$
\Gamma(\phi \rightarrow \rho \pi)=\frac{9 g^{4}\left|\mathbf{q}_{\pi}\right|^{3}}{512 \pi^{5} F_{\pi}^{2}} \epsilon^{2},
$$

where $\mathbf{q}_{\pi}$ is the pion three-momentum in the rest frame of the $\phi$ meson. From the experimental value $\Gamma_{\text {expt }}(\phi \rightarrow \rho \pi) \approx 0.62 \mathrm{MeV}$, we get $|\epsilon|=0.076$, which is in fair agreement with that obtained from the canonical-massmixing model, $\epsilon=-0.058$ [26].

\section{RESULTS AND DISCUSSIONS}

We first consider dilepton production from $\pi+\rho$ annihilation based on the vector meson dominance. The hadronic tensor is given by

$$
\begin{aligned}
h_{\mu \nu}= & 2\left\{\left[q^{2} k^{2}-(q \cdot k)^{2}\right] g_{\mu \nu}+q \cdot k\left(q_{\mu} k_{\nu}+q_{\nu} k_{\mu}\right)-k^{2} q_{\mu} q_{\nu}\right. \\
& \left.-q^{2} k_{\mu} k_{\nu}\right\}\left|F_{\pi \rho}(q)\right|^{2},
\end{aligned}
$$

where $k$ and $q$ are the four-momenta of the $\rho$ meson and the intermediate vector meson, respectively. The form factor $F_{\pi \rho}(q)$ is obtained from the VMD assumption and is given by

$$
\left|F_{\pi \rho}(q)\right|^{2}=\left|F_{\omega}(q)+F_{\phi}(q)\right|^{2} .
$$

In terms of the photon-vector meson coupling constant, $g_{v \gamma}$, and the vector meson width $\Gamma_{V}, F_{V}(q)$ is written as

$$
F_{V}(q)=\frac{g_{v} g_{v \gamma}}{q^{2}-m_{V}^{2}-i m_{V} \Gamma_{V}}
$$

with $g_{\phi}=\epsilon g_{\omega}$.

From Eq. (1), we can then easily obtain the dilepton production rate for the case without including widths of the colliding particles. Since both $\omega$ and $\phi$ mesons can appear as intermediate states of the reaction $\pi+\rho \rightarrow l^{+}+l^{-}$, their interference needs to be included. The result at $T=150 \mathrm{MeV}$ is shown by the dotted curve in Fig. 2. If the interference effect is neglected, then the result is similar to that obtained in Ref. [13]. In the calculation, we have neglected the lepton masses but it is straightforward to include them.

To include particle widths, we assume that the $\rho$ meson spectral function, defined via

$$
\begin{aligned}
\left\langle 0\left|V_{a}^{\mu}(x) V_{b}^{\nu}(0)\right| 0\right\rangle= & -\frac{\delta_{a b}}{(2 \pi)^{3}} \int d^{4} k \theta\left(k^{0}\right) e^{i k \cdot x} \\
& \times\left(g^{\mu \nu}-\frac{k^{\mu} k^{\nu}}{k^{2}}\right) \rho_{V}(k),
\end{aligned}
$$

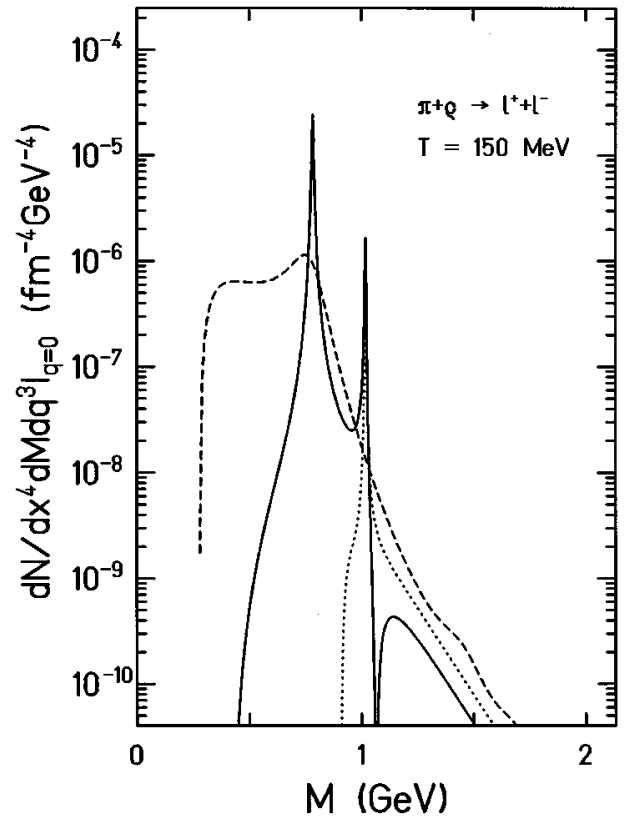

FIG. 2. The dilepton production rate from hot hadronic matter at $T=150 \mathrm{MeV}$. The solid and dotted curves are, respectively, contributions from $\pi+\rho \rightarrow l^{+}+l^{-}$with and without $\rho$ meson width, while the dashed curve is from $\pi+\pi \rightarrow l^{+}+l^{-}$.

has a Breit-Wigner form,

$$
\rho_{V}(k)=\frac{2 m_{\rho} \Gamma_{\rho}}{\left(k^{2}-m_{\rho}^{2}+\Gamma_{\rho}^{2} / 4\right)^{2}+m_{\rho}^{2} \Gamma_{\rho}^{2}},
$$

with $\Gamma_{\rho}=150 \mathrm{MeV}$ being the $\rho$ meson width in free space. It becomes a delta function in the limit $\Gamma_{\rho} \rightarrow 0$, i.e.,

$$
\frac{2 m_{\rho} \Gamma_{\rho}}{\left(k^{2}-m_{\rho}^{2}+\Gamma_{\rho}^{2} / 4\right)^{2}+m_{\rho}^{2} \Gamma_{\rho}^{2}} \rightarrow 2 \pi \delta\left(k^{2}-m_{\rho}^{2}\right) .
$$

For a pion, we assume that

$$
\rho_{\pi}=2 \pi \delta\left(k^{2}-m_{\pi}^{2}\right)
$$

The dilepton production rate is then calculated from Eq. (7). Since the pion spectral function is given by a delta function we are left with only a one-dimensional integral which can be easily evaluated numerically. The result obtained with the inclusion of the rho meson width is shown in Fig. 2 by the solid curve. We see that there is now a large enhancement in the dilepton production rate near the $\omega$ resonance as a result of the extension of the phase space integral. Without including the $\rho$ meson width, it is not possible to have the $\omega$ meson peak in the dilepton spectrum from the $\pi+\rho$ reaction since the threshold value for the invariant mass is above the $\omega$ meson mass. This result is compared with that from the $\pi+\pi$ reaction through the $\rho$ resonance shown by the dashed curve in Fig. 2. We find that the $\omega$ meson peak is above the $\rho$ meson peak from the $\pi+\pi$ reaction due to the very narrow $\omega$ meson width, $\Gamma_{\omega} \sim 8.5 \mathrm{MeV}$. The interference between the $\omega$ and $\phi$ meson intermediate states leads to a reduction in the production rate when $M \geqslant m_{\phi}$ and is most 


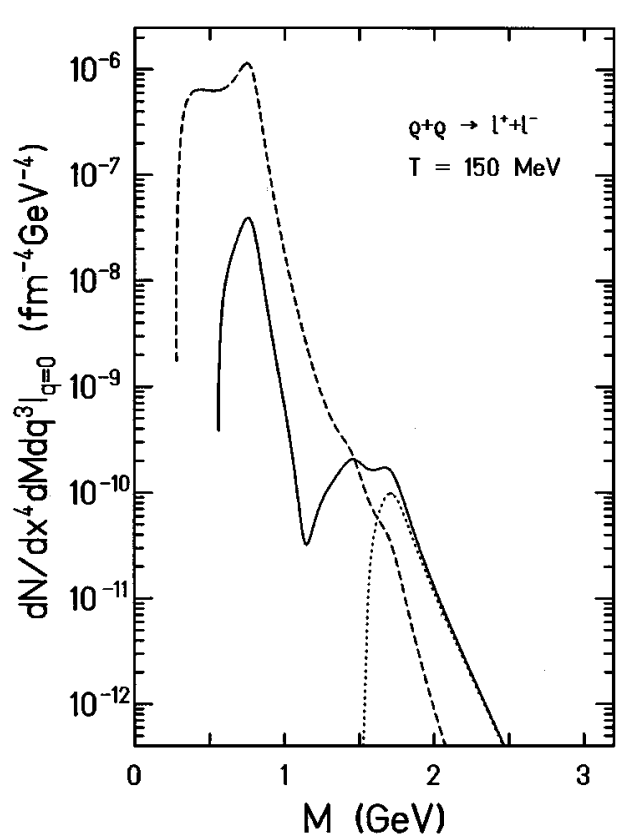

FIG. 3. Same as Fig. 2 for $\rho+\rho \rightarrow l^{+}+l^{-}$.

appreciable at $M \sim m_{\phi}$ as a result of $g_{\omega} \gg g_{\phi}$. However, the effect cannot be observed as it is far below the contribution from the $\pi+\pi$ reaction.

For dileptons from the $\rho+\rho$ reaction, the result at $T=150 \mathrm{MeV}$ including the particle widths is shown in Fig. 3 by the solid curve. We see in the spectrum a $\rho$ peak which is absent in the case without including the particle widths as shown by the dotted curve. However, its magnitude near the resonance is smaller than that from $\pi+\pi$ annihilation (the dashed curve) as a result of the large $\rho$ meson width. In Fig. 4 , the result for the $\pi+a_{1}$ reaction including the $a_{1}$ meson width is shown by the solid curve. It is seen that the $a_{1}$ meson width has only a small effect on the $\pi+a_{1}$ reaction as

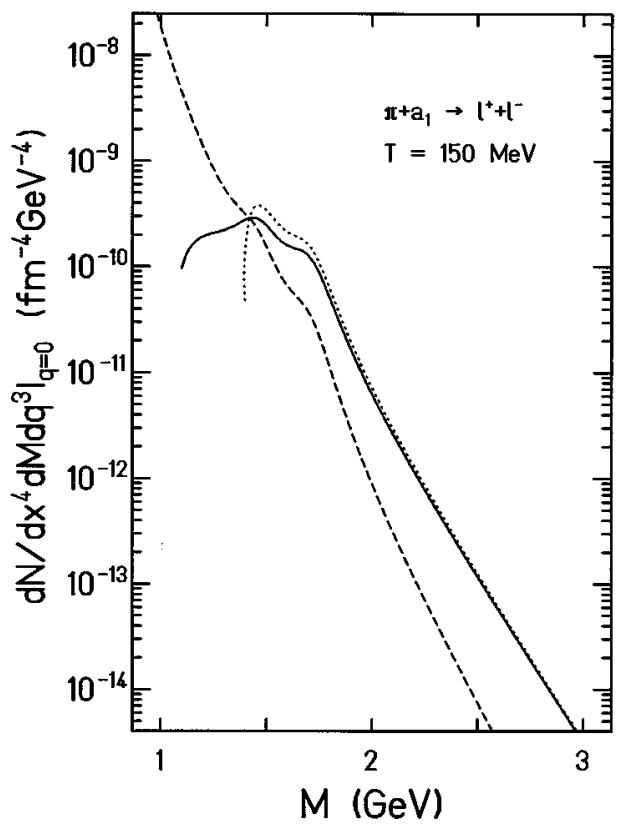

FIG. 4. Same as Fig. 2 for $\pi+a_{1} \rightarrow l^{+}+l^{-}$.

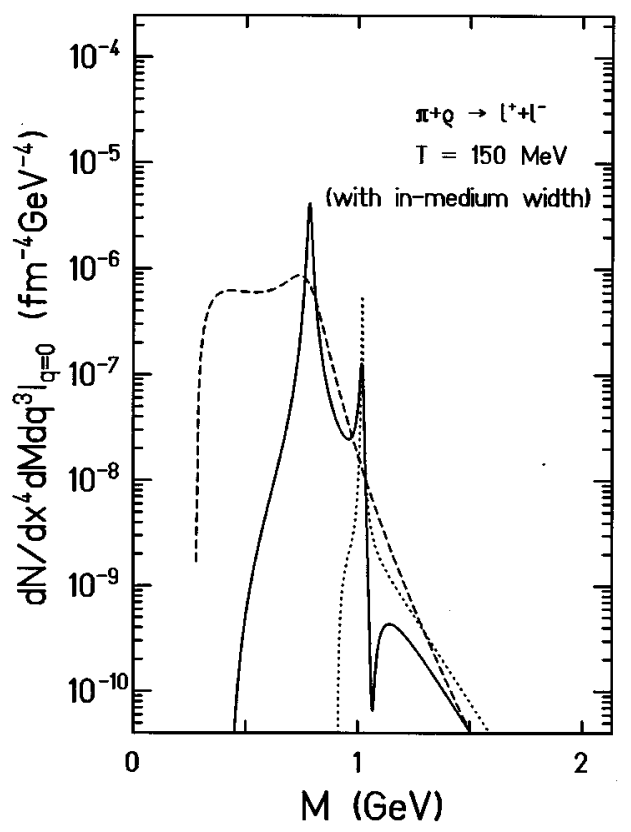

FIG. 5. Same as Fig. 2 with in-medium $\rho, \phi$, and $\omega$ meson widths.

no new resonance appears in the extended phase space when particle widths are included. Compared with the case without including particle widths shown by the dotted curve, there is a small reduction of the production rate near the threshold and also an extension of the production rate into the low invariant mass region.

In hot hadronic matter, mesons change their properties when the temperature of the system approaches the critical value for chiral symmetry restoration and the deconfinement phase transition. In particular, meson widths will be modified by collisions in hot matter. We consider the $\pi+\rho$ reaction in which a sharp peak near the $\omega$ meson resonance appears as a result of its narrow width in free space. Effects due to inmedium widths of the colliding particles are expected to be small because no new resonance is involved. However, the broadening of the resonance in the intermediate state will affect appreciably the dilepton production rate. The dominant contribution to the $\omega$ meson width comes from scattering processes in which the $\omega$ meson interacts with thermal pions through the $b_{1}(1236)$ resonance [27]. To estimate the broadening of its width in hot matter, we use the relation,

$$
\Gamma_{\omega}^{\mathrm{coll}}=\int \frac{d p_{\pi}^{3}}{(2 \pi)^{3}} f_{\pi}\left(E_{\pi}\right) \sigma_{\pi \omega}(M) v_{\mathrm{rel}},
$$

and assume a Breit-Wigner form for the cross section,

$$
\sigma_{\pi \omega}(M)=\mathscr{N} \frac{\pi}{|\mathbf{k}|^{2}} \frac{\Gamma_{b_{1} \rightarrow \pi \omega}^{2}}{\left(M-m_{b_{1}}\right)^{2}+\Gamma_{b_{1}}^{2} / 4},
$$

where $\mathscr{N}$ is the degeneracy factor from the spin and isospin of the colliding particles and resonances. The $\omega$ meson width is found to increase with temperature, and $\Gamma^{\mathrm{coll}} \sim 12 \mathrm{MeV}$ at $T=150 \mathrm{MeV}$ [28].

The effect due to collisional broadening of the intermediate resonance on the $\pi+\rho$ interaction is shown in Fig. 5 by 
the solid curve. We also include the changes in the $\rho$ and $\phi$ meson widths with temperature, which is estimated to be $\Gamma_{\text {coll }} \sim 25 \mathrm{MeV}$ [29] and $10 \mathrm{MeV}$ [30] at $T=150 \mathrm{MeV}$, respectively. We see that the effect of a modified $\omega$ meson width at finite temperature smooths out the peak in the dilepton spectrum. However, there is still a large enhancement of dileptons with invariant masses around the $\omega$ meson when the width of rho meson is included.

\section{CONCLUSION}

Dilepton production from resonance scattering at finite temperature is studied by including widths of the colliding particles. The finite widths increase the phase space for dilepton production and makes it possible that a resonance peak appears below the threshold in the case without particle widths. This effect is significant for $\pi+\rho$ and $\rho+\rho$ reactions in which the extended threshold includes other resonances. However, it has only small effects if there is no resonance appearing in the extended phase space as shown in the $\pi+a_{1}$ reaction. In this case, the production rate is slightly reduced near the threshold and is further extended into lower invariant masses. For the $\pi+\rho$ reaction, the interference between the two possible reactions, $\pi+\rho \rightarrow \omega \rightarrow l^{+}+l^{-}$and $\pi+\rho \rightarrow \phi \rightarrow l^{+}+l^{-}$, leads to a reduction in the dilepton production rate for $M \geqslant m_{\phi}$. However, the effect cannot be observed because of the larger contribution from the $\pi+\pi$ reaction.

Particle widths are modified in hot matter, and this also affects the dilepton production rate. Again, the effect due to changes in the widths of the colliding particles is small unless new resonances appear in the extended phase space. When thermal effects on the widths of intermediate resonances are included, we obtain a reduction of the resonance peak in the dilepton spectrum as shown in the $\pi+\rho$ reaction. However, there is still a significant enhancement of dileptons with invariant masses near the vector meson resonance compared with the case without including the widths of the colliding particles.

In future studies of dilepton production from nucleusnucleus collisions, one should thus include both finite particle widths and the medium modification of hadron properties. This is necessary for identifying the signatures of the quark-gluon plasma and the precursor phenomena related to the phase transition from the hadronic background. We expect that for photon production from hot dense matter there will be similar effects, and we plan to investigate this in the future.

\section{ACKNOWLEDGMENTS}

This work was supported in part by the National Science Foundation under Grant No. PHY-9212209 and the Welch Foundation under Grant No. A-1110. The support of C.M.K. by the Humboldt Research Foundation is also gratefully acknowledged, and he would like to thank Ulrich Mosel of the University of Giessen for the warm hospitality.
[1] For a recent review see Quark Matter '93, Nucl. Phys. A556, 1 (1994).

[2] L. McLerran, Rev. Mod. Phys. 58, 1021 (1986).

[3] E. L. Feinberg, Nuovo Cimento A 34, 39 (1976); E. V. Shuryak, Sov. J. Nucl. Phys. 28, 408 (1978).

[4] L. McLerran and T. Toimela, Phys. Rev. D 31, 545 (1985).

[5] R. Pisarski, Phys. Lett. 110B, 155 (1982).

[6] K. Kajantie, J. Kapusta, L. McLarren, and A. Mekjian, Phys. Rev. D 34, 2746 (1986).

[7] C. M. Ko and L. H. Xia, Phys. Rev. Lett. 62, 1595 (1989); M. Asakawa, C. M. Ko, and P. Lévai, ibid. 70, 398 (1993); M. Asakawa and C. M. Ko, Phys. Lett. B 322, 33 (1994).

[8] T. Hatsuda, Y. Koike, and Su H. Lee, Phys. Rev. D 47, 1225 (1993).

[9] J. Kapusta, P. Lichard, and D. Seibert, Phys. Rev. D 44, 2774 (1991).

[10] L. Xiong, E. V. Shuryak, and G. E. Brown, Phys. Rev. D 46, 3798 (1992).

[11] Chungsik Song, Phys. Rev. C 47, 2861 (1993).

[12] K. Haglin, Phys. Rev. C 50, 1688 (1994).

[13] C. Gale and P. Lichard, Phys. Rev. D 49, 3338 (1994).

[14] C. Song, C. M. Ko, and C. Gale, Phys. Rev. D 50, R1827 (1994).

[15] J. J. Neumann, D. Seibert, and G. Fai, Phys. Rev. C 51, 1460 (1995); A. Dumitru, U. Katscher, J. A. Marhun, H. Stöcker, W. Greiner, and D. Rischke, ibid. 51, 2166 (1995).

[16] D. Srivastava, B. Sinha, and C. Gale, McGill University report, 1995.
[17] C. Song, S. H. Lee, and C. M. Ko, Phys. Rev. C 52, R476 (1995).

[18] C. Dominguez, M. Loewe, and J. Rozowsky, Phys. Lett. B 335, 506 (1994).

[19] D. Kharzeev and H. Satz, Phys. Lett. B 340, 167 (1994).

[20] C. M. Ko, L. H. Xia, and P. J. Siemens, Phys. Lett. B 246, 333 (1990).

[21] S. Weinberg, Physica A 96, 327 (1979).

[22] N. M. Kroll, T. D. Lee, and B. Zumino, Phys. Rev. 157, 1376 (1967).

[23] Chungsik Song, Phys. Rev. D 48, 1375 (1993).

[24] J. J. Sakurai, Currents and Mesons (Chicago Press, Chicago, 1969).

[25] E. Witten, Nucl. Phys. B233, 432 (1983); Ö. Kaymakcalan, S. Rajeev, and J. Schechter, Phys. Rev. D 30, 594 (1984).

[26] H. Gomm, Ö. Kaymakcalan, and J. Schechter, Phys. Rev. D 30, 2345 (1984).

[27] K. Haglin, Nucl. Phys. A584, 719 (1995).

[28] The collision rate for $\omega$ meson is reduced by a factor of 4 compared with that in Ref. [12] as we have used $\Gamma_{\pi+\omega \rightarrow b_{1}}=(1 / 2) \Gamma_{\text {total }}$.

[29] The collision rate for the $\rho$ meson is estimated in the same way as for the $\omega$ meson. We assume that the dominant contribution comes from the $a_{1}$ resonance. More detailed calculations show that the $\rho$ meson width increases by about $40 \mathrm{MeV}$ at $T=150 \mathrm{MeV}$ [27].

[30] C. M. Ko and D. Seibert, Phys. Rev. C 49, 2198 (1994). 